\title{
Interaction of RRF and EF-G from E. coli and T. thermophilus with ribosomes from both origins- insight into the mechanism of the ribosome recycling step
}

\author{
V. SAMUEL RAJ ${ }^{1,2}$ HIDEKO KAJI, $^{2}$ and AKIRA KAJI ${ }^{1,3}$ \\ ${ }^{1}$ Department of Microbiology, School of Medicine, University of Pennsylvania, Philadelphia, Pennsylvania 19104, USA \\ ${ }^{2}$ Department of Biochemistry and Molecular Pharmacology, Jefferson Medical College, Thomas Jefferson University, \\ Philadelphia, Pennsylvania 19107, USA \\ ${ }^{3}$ Creative Biomedical Research Institute, Philadelphia, Pennsylvania 19147, USA
}

\begin{abstract}
Ribosome recycling factor (RRF), elongation factor-G (EF-G), and ribosomes from Thermus thermophilus (tt-) and Escherichia coli (ec-) were used to study the disassembly mechanism of post-termination ribosomal complexes by these factors. With tt-RRF, ec-EF-G can release bound-tRNA from ec-model post-termination complexes. However, tt-RRF is not released by ec-EF-G from ec-ribosomes. This complex with tt-RRF and ec-ribosomes after the tRNA release by ec-EF-G is regarded as an intermediate of the disassembly reaction. Not only tt-RRF, but also mRNA, cannot be released from ec-ribosomes by tt-RRF and ec-EF-G. These data suggest that the release of RRF from ribosomes is coupled or closely related to the release of mRNA during disassembly of post-termination complexes. With tt-ribosomes, ec-EF-G cannot release ribosome-bound ec-RRF even though they are from the same species, showing that proper interaction of ec-RRF and ec-EF-G does not occur on tt-ribosomes. On the other hand, in contrast to a published report, tt-EF-G functions with ec-RRF to disassemble ec-post-termination complexes. In support of this finding, tt-EF-G translocates peptidyl tRNA on ec-ribosomes and catalyzes ec-ribosome-dependent GTPase, showing that tt-EF-G has in vitro translocation activity with ec-ribosomes. Since tt-EF-G with ec-RRF can release tRNA from ec-post-termination complexes, the data are consistent with the hypothesis that the release of tRNA by RRF and EF-G from post-termination complexes is a result of a translocation-like activity of EF-G on RRF.
\end{abstract}

Keywords: ribosome; recycling; EF-G; RRF; post-termination; translation

\section{INTRODUCTION}

Ribosome recycling factor (RRF) with elongation factor-G (EF-G) disassembles the post-termination ribosomal complexes into ribosomes, mRNA, and deacylated tRNA (Janosi et al. 1996; Kaji et al. 2001). RRF is essential for prokaryotes (Janosi et al. 1994) and is a heat-shock protein (TeixeiraGomes et al. 2000). The bacterial cellular RRF concentration is elevated during the infection of animals by that bacteria (Lowe et al. 1998), and the amount of antibacterial RRF antibodies is high in some infected animals (Vizcaino et al. 1996; Cassataro et al. 2002), suggesting that RRF may play an important role in bacterial pathogenesis. RRF is an essential protein in prokaryotes (Janosi et al. 1994) and

Reprint requests to: Akira Kaji, Department of Microbiology, School of Medicine, University of Pennsylvania, Room 203B, Johnson Pavilion, 3610 Hamilton Walk, Philadelphia, PA 19104, USA; e-mail: kaji@mail.med. upenn.edu; fax: (215) 573-2221.

Article published online ahead of print. Article and publication date are at http://www.rnajournal.org/cgi/doi/10.1261/rna.7201805. eukaryotes also (Rolland et al. 1999; Teyssier et al. 2003). The biological importance of RRF was further supported by isolation of a number of RRF mutants (Janosi et al. 1998, 2000; Fujiwara et al. 1999, 2001) that have lethal effects. In the absence of RRF, ribosomes are not released from mRNA and start unscheduled translation downstream of the termination codon (Janosi et al. 1998). These ribosomes translating downstream of the termination codon eventually reach the end of mRNA and are transferred to tmRNA (Hirokawa et al. 2004). Ribosomes isolated from these RRFlacking extracts were inactive for translation of conventional mRNA, but active for translation of leaderless mRNA (Moll et al. 2004). Since leaderless mRNA does not require subunit dissociation while conventional mRNA does, these results are consistent with the recent observation that RRF is involved in dissociation of ribosomes into subunits (G. Hirokawa, R.M. Nijman, S.V. Raj, H. Kaji, K. Igarashi, and A. Kaji, in prep.).

For the disassembly reaction, EF-G and RRF have to be present simultaneously (Hirashima and Kaji 1973). In other words, these factors have to function together. RRF binds to 
the A/P-site of the ribosome (Hirokawa et al. 2002a; Lancaster et al. 2002; Agrawal et al. 2004), but an additional site for RRF ribosomal binding has been reported (Kiel et al. 2003). Our recent studies with fluorescent-labeled RRF confirmed the existence of the second RRF-binding site on the ribosome (Seo et al. 2004). It has been proposed that RRF is moved from the A/P-site to this second site by EF-G, resulting in the release of tRNA from the ribosome (Hirokawa et al. 2002b; Kiel et al. 2003). This is reminiscent of conventional translocation, in which tRNA is released by EF-G (Ishitsuka et al. 1970). Ribosome-bound RRF is released by EF-G (Kiel et al. 2003), in much the same way tRNA is released by EF-G (Ishitsuka et al. 1970). This observation was confirmed recently (Fujiwara et al. 2004). The structure of RRF elucidated by crystallography (Selmer et al. 1999) as well as by NMR (Yoshida et al. 2001) is similar to that of tRNA. The structure was confirmed by three other laboratories (Kim et al. 2000; Toyoda et al. 2000; Nakano et al. 2003). The structural similarity of RRF to tRNA may help RRF bind to the 70S ribosome at the intersubunit space (space between the $30 \mathrm{~S}$ and $50 \mathrm{~S}$ subunits) and then move within the intersubunit space similarly to tRNA.
In this paper, we took advantage of the fact that tt-RRF can function in Escherichia coli having defective ec-RRF (Fujiwara et al. 1999) and examined the mechanism of disassembly of the post-termination complexes using factors from Thermus thermophilus and E. coli. We show that ttRRF remains on ec-ribosomes after releasing tRNA from the model post-termination complexes. We consider that this ribosomal complex with tt-RRF is an intermediate during the disassembly reaction by RRF and EF-G. Our observation suggests that the release of mRNA by EF-G is coupled or closely related to the release of RRF from ribosomes during the disassembly reaction.

\section{RESULTS}

\section{Disassembly of ec-model post-termination complexes by various combinations of RRF and EF-G from $E$. coli and T. thermophilus: Species-specific interaction of these factors is only one way}

In Figure $1 C$ we show that $T$. thermophilus RRF (tt-RRF) was not able to function with E. coli EF-G (ec-EF-G) for disassembly of ec-model post-termination complexes, as

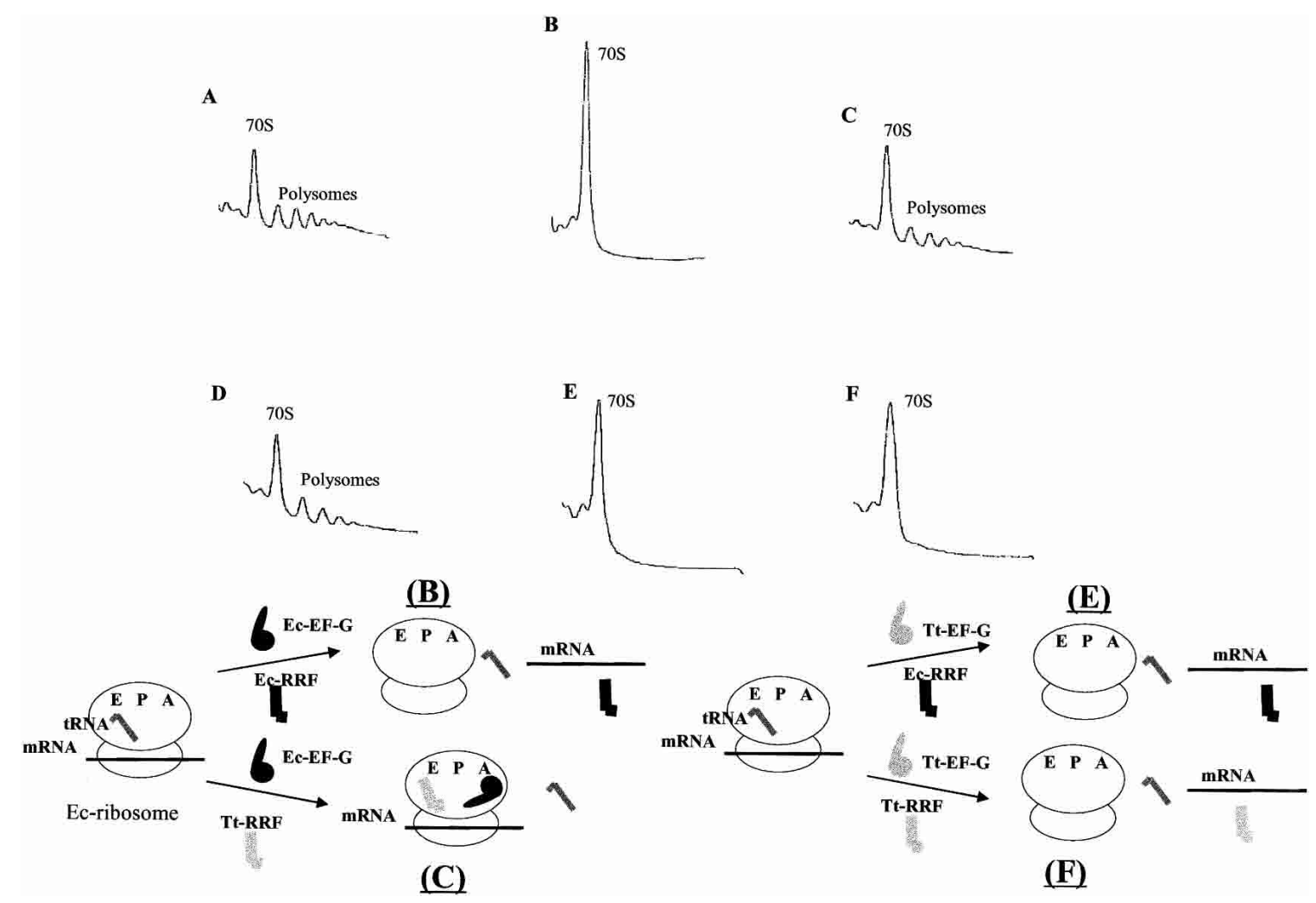

FIGURE 1. Species specificity of RRF and EF-G is only one-way. $(A-C)$ The reaction mixture $(275 \mu \mathrm{L})$ contained $4.9 \mu \mathrm{M}$ ec-EF-G, $363 \mu \mathrm{M}$ GTP, $50 \mu \mathrm{M}$ puromycin, $0.6 \mathrm{~A}_{260}$ unit $(0.05 \mu \mathrm{M})$ polysomes and RRF where indicated. (A) No RRF; (B) $0.72 \mu \mathrm{M}$ of ec-RRF; and $(C) 0.72 \mu \mathrm{M}$ of tt-RRF. $(D-F)$ As in $A-C$ with $50 \mathrm{pmol}(0.18 \mu \mathrm{M})$ of tt-EF-G instead of ec-EF-G. $(D)$ No RRF; $(E) 0.18 \mu \mathrm{M}$ of ec-RRF; and $(F) 0.18 \mu \mathrm{M}$ of tt-RRF. After $15 \mathrm{~min}$ at $30^{\circ} \mathrm{C}$, the sedimentation profile $\left(40 \mathrm{~K} \mathrm{rpm}\right.$ for $75 \mathrm{~min}$ at $4^{\circ} \mathrm{C}$ through $15 \%-30 \%$ sucrose gradient in Beckman rotor SW 50.1$)$ was analyzed by an ISCO UA-6 Spectrophotometer at $\mathrm{A}_{254}$. Sedimentation is from left to right. Conversion of polysomes to monosomes is a reflection of RRF activity. The pictures underneath the sedimentation profiles represent the reactions leading to the results described in $B, C, E$, and $F$. Components of the reactions are labeled. The symbols representing each component are the same for other pictures shown in other figures. 
measured by the conversion of puromycin-treated ec-polysomes to monosomes (a method used for the routine assay of RRF) (Hirashima and Kaji 1972a). On the other hand, homologous combination of RRF and EF-G from E. coli and T. thermophilus resulted in complete conversion of ec-polysomes to monosomes, indicating that the release of mRNA took place (Fig. 1, cf. B and A, F and D). These results are expected, based on previous work from other laboratories (Rao and Varshney 2001; Ito et al. 2002).

However, results contradictory to those of Ito et al. (2002) were obtained when we combined ec-RRF and ttEF-G. This is the forbidden combination and is not supposed to function, but ec-RRF functioned perfectly well with tt-EF-G (Fig. 1, cf. E and D). The extent of the reaction was just as good as that with homologous combination of RRF and EF-G from the same species (Fig. 1F). Figure 2A shows that almost an equally small amount of tt-EF-G functions with ec-RRF in the disassembly reaction. A Lineweaver-Burke plot (Fig. 2B) shows that the $K_{\mathrm{m}}$ value for $\mathrm{tt}-\mathrm{EF}-\mathrm{G}$ is $4 \mathrm{nM}$, equal to that of ec-EF-G (4 nM). We conclude that tt-EF-G functions with ec-RRF. We should point out that this discrepancy does not affect the major conclusion of this paper. In fact, the important conclusion that combination of tt-RRF and ec-EF-G gives an intermediate of the disassembly reaction was based on the original finding of Ito et al. (2002) in which the combination of these two factors does not function for the complete disassembly.

Although we observed that tt-RRF does not function with ec-EF-G for the release of mRNA, even this specificity is dependent on the quantity of RRF as shown in Figure 3. In this experiment, the reaction mixture contained a constant amount of ec-EF-G, and we added increasing amounts of tt-RRF. An excess of tt-RRF, up to $18.2 \mu \mathrm{M}$, functioned

(A)
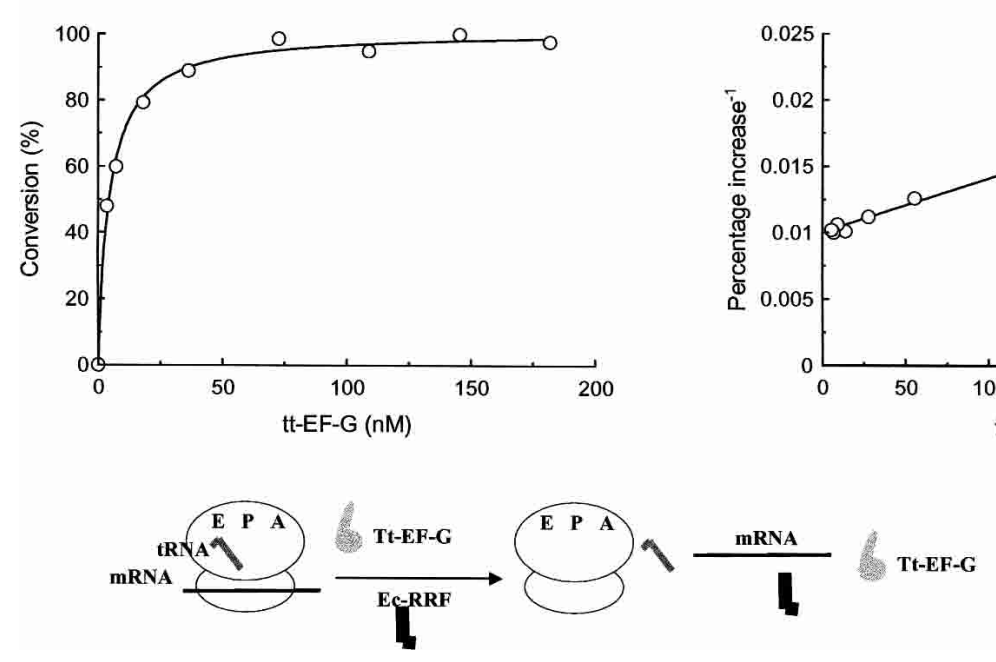

partially for disassembly, indicating that tt-RRF may function with ec-EF-G at low efficiency. Since the $\mathrm{K}_{\mathrm{d}}$ of ec-RRF to ec-model post-termination complexes is $0.03 \mu \mathrm{M}$ (Kiel et al. 2003), the efficiency of tt-RRF is about 1/600 of that of ec-RRF. Nevertheless, tt-RRF functioned and the activity of tt-RRF we observed must be due to tt-RRF because the contamination by ec-RRF, if any, was $<0.01 \%$ as examined by specific antibodies to ec-RRF (data not shown). This is consistent with the observation that very small, minute colonies grow when one examines complementation of ecRRF amber mutants by tt-RRF (Fujiwara et al. 1999). Since physiological concentration of RRF in vivo is $\sim 20 \mu \mathrm{M}$ (Andersen et al. 1999), results with $18 \mu \mathrm{M}$ tt-RRF may even be significant.

\section{The one-way species-specific interaction between EF-G and RRF for the release of RRF is the same for the release of $\mathrm{mRNA}$-isolation of intermediate of ribosome recycling process}

In E. coli, ec-RRF is released from ribosomes by ec-EF-G. This is a result of RRF being moved by EF-G from its high-affinity A/P-site to a second lower-affinity site. It was proposed that RRF performs the final act, the release of mRNA and itself from ribosomes, at this second site (Kiel et al. 2003). Therefore, the release of RRF from ribosomes is a vital part of the disassembly reaction.

In the experiment described in Figure 4A, tt-RRF or ecRRF was bound to vacant ec-ribosomes, and the resulting complexes were isolated. The complexes were then treated with ec- or tt-EF-G, and the release of RRF was examined. As described recently, the binding site for EF-G after translocation overlaps with the A/P-site-bound RRF (Agrawal et

(B)

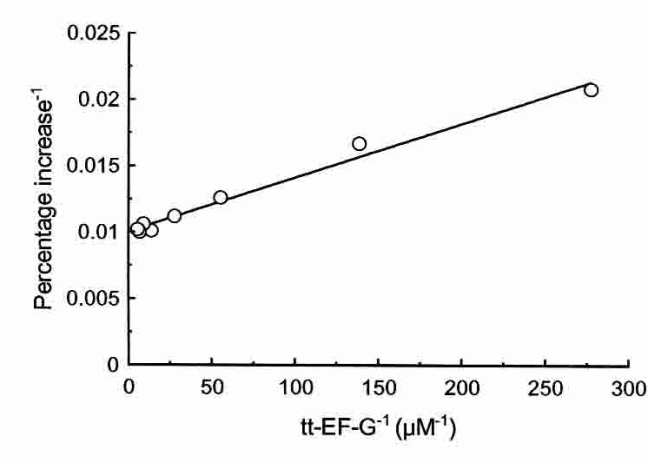
t-EF-G

FIGURE 2. tt-EF-G functions with ec-RRF as efficiently as ec-EF-G. (A) Dose response curve of tt-EF-G for disassembly of the model post-termination complexes as performed in Figure 1. (B) Lineweaver-Burke plot of $A$. The $K_{\mathrm{m}}$ for tt-EF-G from this plot is $4 \mathrm{nM}$. Here $0.05 \mu \mathrm{M}$ polysome and $1.96 \mu \mathrm{M}$ ec-RRF were used. The pictures underneath the figure represent the disassembly shown in the figure. 

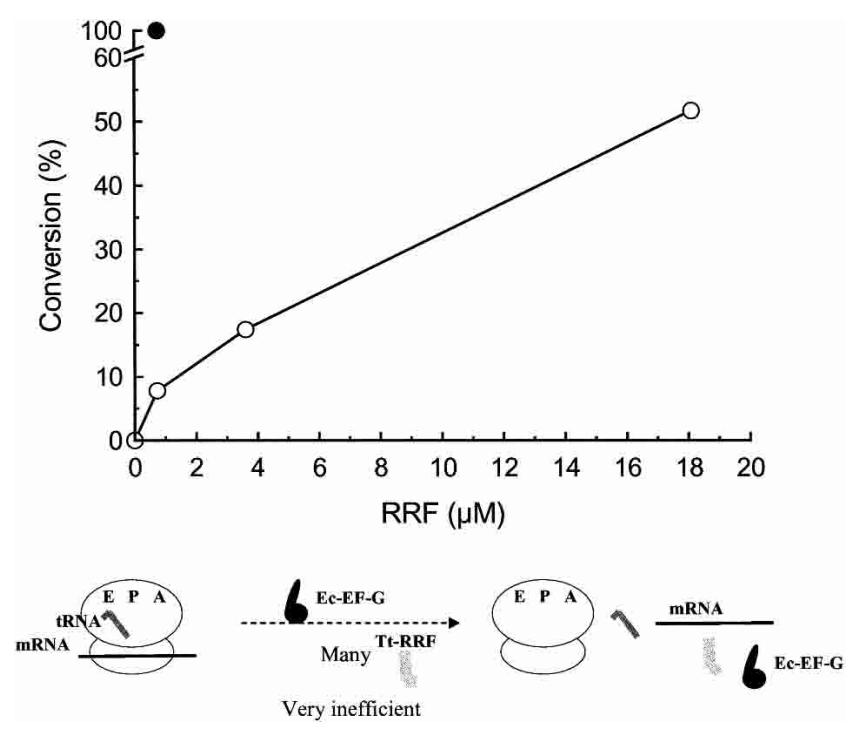

FIGURE 3. High concentrations of tt-RRF function with ec-EF-G for the disassembly reaction. The reaction mixture $(275 \mu \mathrm{L})$ contained 4.9 $\mu \mathrm{M}$ ec-EF-G, $363 \mu \mathrm{M}$ GTP, $50 \mu \mathrm{M}$ puromycin, $0.6 \mathrm{~A}_{260}$ unit polysomes $(0.05 \mu \mathrm{M})$, and various amounts of tt-RRF (open circles) or ec-RRF (filled circle) in buffer X. The conversion of polysomes into monosomes was determined as in Figure 1 and expressed as a percentage of polysomes converted to monosomes by RRF and EF-G. The conversion of polysomes into monosomes by $200 \mathrm{pmol}$ ec-RRF $(0.72$ $\mu \mathrm{M})$ under the same condition was considered a $100 \%$ conversion. The pictures underneath the figure represent the disassembly shown in the figure. The dotted arrow indicates inefficient reaction by this combination of RRF and EF-G.

al. 2004). Therefore, the bound RRF is pushed to the second site and released. As shown in this figure, release of RRF is dependent on the amount of EF-G added. The reason that one does not observe complete release of RRF is that not all well-washed ribosomes bind EF-G, while most of them can bind RRF (Kiel et al. 2003). Most importantly, it shows that tt-RRF is not released by ec-EF-G (open squares). This is the only combination that does not allow for the release of mRNA measured by conversion of polysomes (model posttermination complex) to monosomes (see Fig. 1C). It should be noted that the combinations ec-RRF/ec-EF-G (Fig. 1B), ec-RRF/tt-EF-G (Fig. 1E), and tt-RRF/tt-EF-G (Fig. 1F) function well for the release of mRNA, as measured by the disassembly of model post-termination complexes. These data are consistent with the notion that the step in which RRF is released may also be the step in which mRNA is released.

Similar one-way specificity was observed for the release of RRF from ec-model post-termination complexes (Fig. $4 \mathrm{~B})$. It should be noted that tt-RRF is not released by ecEF-G under these conditions (see Fig. 5), indicating that tt-RRF can be caught after it released tRNA. We therefore conclude that the results obtained with vacant ribosomes are repeatable with more physiological model post-termination complexes, indicating that the experiments using vacant ribosomes are physiologically relevant.
All studies described thus far were carried out with ecribosomes. As shown in Figure 4C, when one uses tt-ribosomes, the specificity rule is quite different. tt-Ribosomebound ec-RRF cannot be released by ec-EF-G (open symbols). This is a combination that is supposed to function just as the combination of tt-RRF and tt-EF-G worked with ec-ribosomes. It appears that the species-specific interaction of RRF and EF-G must depend on the source of ribosomes and, depending on the source of ribosomes, even the combination of RRF and EF-G from the same species does not function. It is noted that the combination of tt-RRF and tt-EF-G functioned as expected. Again, the combination of ec-RRF and tt-EF-G functioned even with tt-ribosomes. This is a similar situation to ec-ribosomes, where the same combination functioned (Fig. 4A,B). We consider that, under these conditions, tt-RRF is moved on tt-ribosomes but remained at that tt-ribosomal site because ec-EF-G cannot release tt-RRF from the tt-ribosome.

\section{Species-specific interaction of EF-G and RRF is not required for $T R N A$ release from ec-model post-termination complexes by RRF and EF-G-tt-RRF remains on ec-ribosomes after it releases tRNA}

With model post-termination complexes, we have shown that tRNA is released prior to the release of mRNA (Hirokawa et al. 2002b). In Figure 5, we examined if the release of tRNA requires species-specific interactions between EF-G and RRF. As shown in this figure, tt-RRF, together with ec-EF-G, functions perfectly well to release ec-ribosomebound tRNA. Despite the inability of this combination to function in the release of mRNA, tt-RRF functioned to release all ribosome-bound tRNA from ec-ribosomes with efficiency comparable to that of ec-RRF. We should point out that under these experimental conditions, tt-RRF remains on ec-ribosomes (see open squares in Fig. 4C). As pointed out in Figure 4, we consider that, under these conditions, tt-RRF is moved on ec-model post-termination complexes to release tRNA but remained at that site because ec-EF-G cannot release tt-RRF from the ec-ribosome.

\section{tt-EF-G catalyzes GTP hydrolysis and translocation with ec-ribosomes like ec-EF-G but at a much slower rate}

It has been shown that tt-EF-G does not complement (Ito et al. 2002) temperature-sensitive ec-EF-G (Hou et al. 1994a). We confirmed this finding (data not shown). We showed that ec-RRF functions with tt-EF-G (Fig. 1). Why then can't tt-EF-G complement a temperature-sensitive ec-EF-G mutant in vivo? To answer this question, we examined typical EF-G-dependent reactions, GTPase (Rohrback and Bodley 1976) and translocation, with ec-ribosomes. As shown in Figure 6A, GTPase activity is proportionally dependent on both tt-EF-G and ec-EF-G, but the efficiency of tt-EF-G 
A

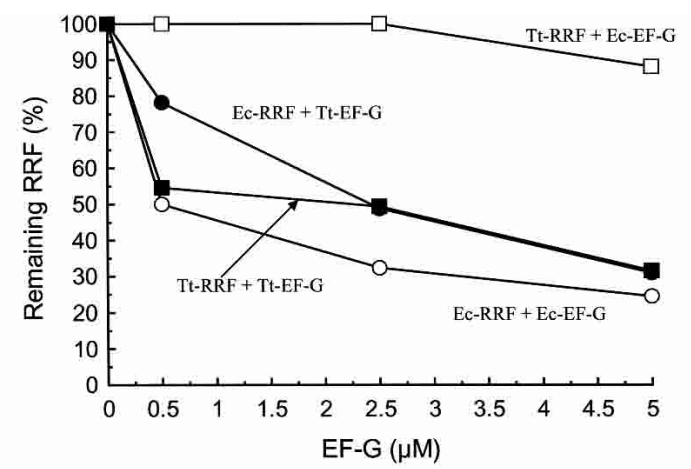

B
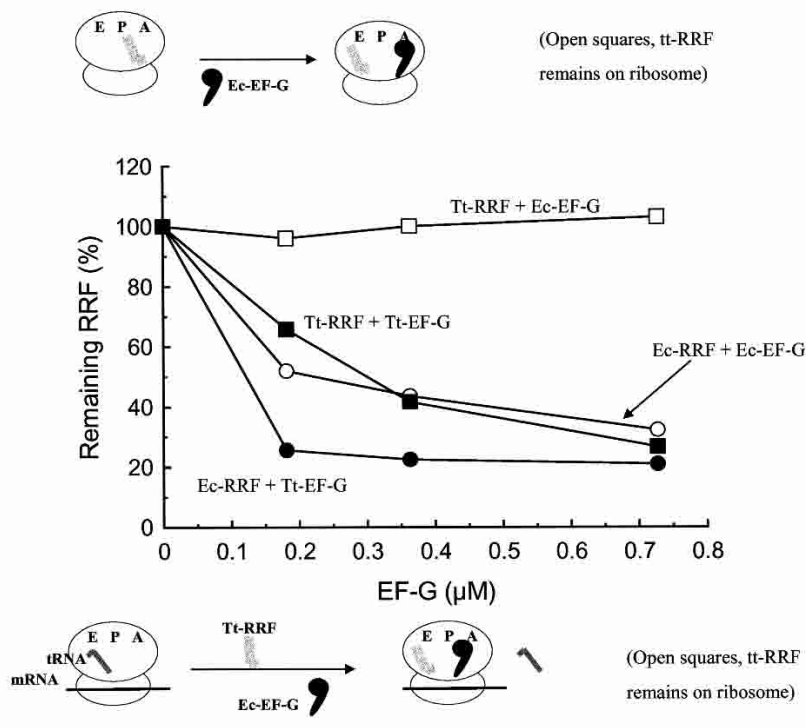

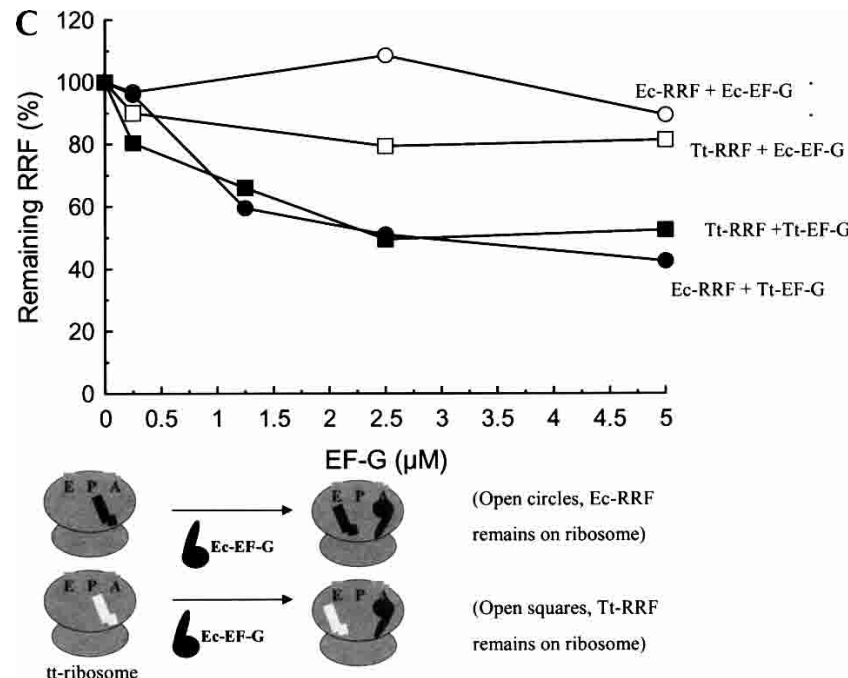

Post termination complex

FIGURE 4. Specific interaction of RRF with EF-G for the release of RRF from ribosomes is the same as that for the release of mRNA. (A) ec-RRF is released by both ec- and tt-EF-G, but tt-RRF is released only by tt-EF-G from complexes of vacant ec-ribosomes and RRF. (Open circles) ec-RRF complexes and ec-EF-G; (closed circles) ec-RRF complexes and tt-EF-G; (open squares) tt-RRF and ec-EF-G; (closed squares) tt-RRF and tt-EF-G. $\mathrm{RRF} /$ ribosome complexes, prepared by incubating $0.25 \mu \mathrm{M}$ ribosomes and $2.5 \mu \mathrm{M} R \mathrm{R}$, were isolated and incubated with the factors as indicated in buffer $\mathrm{R}$ for $10 \mathrm{~min}$ at $35^{\circ} \mathrm{C}$. The pictures underneath the figure show that tt-RRF remains on the ribosome in the presence of ec-EF-G (open squares in the figure). (B) The same as $A$ except model post-termination complexes were used. Here, $0.05 \mu \mathrm{M}$ ribosomes (polysomes) and 0.18 $\mu \mathrm{M}$ RRF were incubated at $30^{\circ} \mathrm{C}$ for $15 \mathrm{~min}$ (identical to the routine RRF assay). The symbols are the same as in $A$. The pictures underneath the figure show that tt-RRF remains on the post-termination complex in the presence of ec-EF-G (open squares in the figure). (C) RRF release by EF-G from complexes of vacant tt-ribosomes and RRF. The ribosomes were from $T$. thermophilus. The symbols are the same as in $A$. Complexes were prepared by incubating $0.25 \mu \mathrm{M}$ ribosomes and $3.75 \mu \mathrm{M} \mathrm{RRF}$ at $35^{\circ} \mathrm{C}$ for $10 \mathrm{~min}$ in buffer $\mathrm{R}$. The pictures underneath the figure show that ec- or tt-RRF remains on the tt-ribosome in the presence of ec-EF-G (open circles and squares in the figure).

with ec-ribosomes was $\sim 8 \%-9 \%$ of ec-EF-G. In the inset figure, we show again that tt-EF-G has a significant GTPase activity dependent on the amounts of ec-ribosomes. However, the difference between these two EF-Gs becomes more prominent when the ribosome concentrations are increased. We estimate that the rate of GTP hydrolysis by 3 $\mu \mathrm{M}$ tt-EF-G and $0.4 \mu \mathrm{M}$ ec-ribosomes was at least 250 -fold less than that by $0.4 \mu \mathrm{M}$ ec-EFG and $0.4 \mu \mathrm{M}$ ec-ribosomes.

Similarly, tt-EF-G has significant translocation activity with ec-ribosomes. In Figure 6B, translocation of oligophenylalanyl tRNA (Suzuka and Kaji 1967) from the A/Psite (Noller et al. 2002) to the P/P-site was studied with ttas well as ec-EF-G on ec-ribosomes programmed with poly $(\mathrm{U})$. When peptidyl tRNA is translocated from the A/P- site to the $\mathrm{P} / \mathrm{P}$-site, it becomes accessible to puromycin to form peptidyl puromycin (Yarmolinsky and de la Haba 1959). Under the experimental conditions used in this figure, the translocation is proportional to the amount of ecEF-G added (see the inset figure). Figure $6 \mathrm{~B}$ shows that the initial rate with tt-EF-G is about one-third of that with ec-EF-G using the same amount of the enzyme. However, in the inset figure, we show that increasing amounts of ec-EF-G increased the reaction rate, while tt-EF-G did not do so.

\section{DISCUSSION}

We believe that the studies with tt-RRF described in this paper have physiological significance because tt-RRF func- 

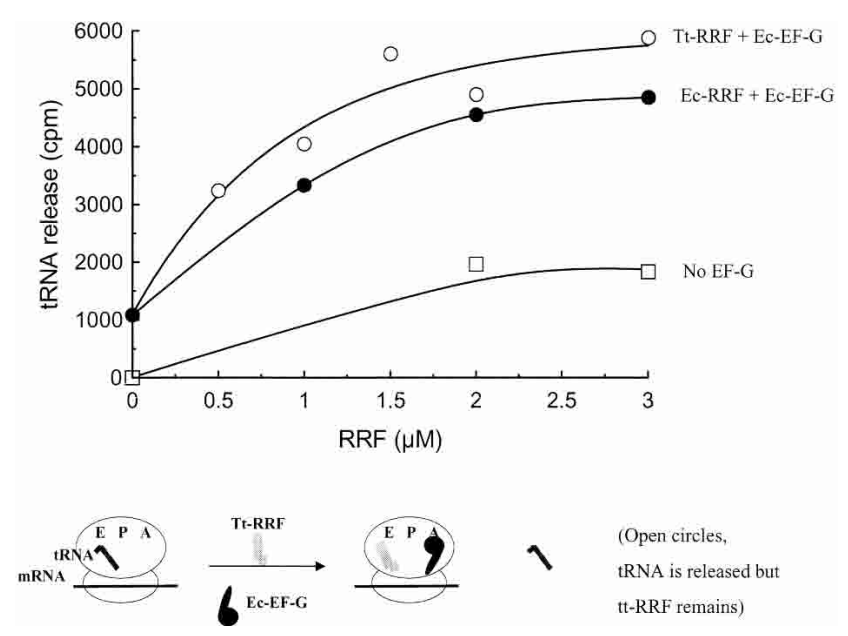

FIGURE 5. tt-RRF can release ec-ribosome-bound tRNA with ec-EFG. Release of tRNA from model post-termination complexes was carried out by EF-G and RRF as described in Materials and Methods. The released tRNA is expressed as the amount of ${ }^{14} \mathrm{C}$-amino-acid-labeled aminoacyl tRNA obtained after aminoacylating the tRNA released from post-termination complexes. The total ribosome-bound tRNA is $7184 \mathrm{cpm}$, corresponding to $\sim 50 \mathrm{pmol}$ of tRNA (see Materials and Methods). Radioactivity is plotted against the amounts of RRF added. (Open circles) tt-RRF and ec-EF-G; (closed circles) ec-RRF and ecEF-G; (open squares) background release of tRNA by tt-RRF in the absence of EF-G. The amounts released $(1376 \mathrm{cpm})$ in the absence of factors have been subtracted from the observed values. The pictures underneath the figure show that tt-RRF remains on the post-termination complex after the release of tRNA in the presence of ec-EF-G (open squares in the figure).

tions with ec-ribosomes in vivo (Ito et al. 2002) and in vitro (this paper) if tt-EF-G is provided. On this basis, the binding site after it is moved by ec-EF-G is assumed to be the same binding site of ec-RRF after it is moved by ec-RRF. We should emphasize, however, that a potential problem is the combination of heterologous factors/ribosomes, and that they originate from a mesophilic and a thermophilic bacterium. Exact identity of the binding sites of ec-RRF and tt-RRF should be compared with more biophysical approaches such as solving the crystal structure of the ribosome with tt-RRF and fluorescence resonance energy transfer (FRET) studies with ec-RRF. Such studies are in progress. With this reservation in mind, Figure 7 summarizes our hypothesis based on the findings in this paper and information accumulated from other studies. Complex I is the ec-post-termination complex with tt-RRF at the A/Psite, unesterified tRNA at the P/E-site, and EF-G at the pre-translocation site. The most significant finding from the present study is that tt-RRF remains on ec-ribosomes (Fig. $4 \mathrm{C}$, open squares) after it releases tRNA with the help of ec-EF-G (Fig. 5, open circles; complex II of Fig. 7). The complex II remains as such without proceeding to complex III because ec-EF-G cannot release tt-RRF from the ribosome.

Under the conditions described in Figure 5, we propose that tt-RRF is at the second site from which it will perform the final act, the release of mRNA if tt-EF-G is given. We suggest that this site is the same site where ec-RRF is moved to and released from the ribosomes during normal disassembly of the post-termination complexes. This hypothesis is supported by the fact that tt-RRF functions in E. coli that have defective ec-RRF (Fujiwara et al. 2001). Therefore, binding of tt-RRF to ec-ribosomes described here is identical to that of ec-RRF. The difference in the behavior is that $\mathrm{tt}-\mathrm{RRF}$ requires $\mathrm{tt}-\mathrm{EF}-\mathrm{G}$ to release it from the ec-ribosome.

Our recent data show that this second ribosomal site for RRF overlaps with the E-site (V. Raj, H. Kaji, and A. Kaji, in prep.). We determined this site to be the E-site by exactly the same procedure we used to determine the first RRFbinding site to be the A/P-site (Hirokawa et al. 2002a). We used competition for this site with $\mathrm{N}$-acetylphenylalanyl tRNA (specific to the P-site) and unesterified tRNA (binds to the $\mathrm{P}$ - and E-site). This conclusion is verified by more direct method of visualizing RRF on the ribosome by hydroxyl radical probing (Lancaster et al. 2002) and electron microscopy (Agrawal et al. 2004), indicating that this method is valid. The presence of the second RRF-binding site was recently confirmed by the use of fluorescent-labeled RRF (Seo et al. 2004). In fact, in the presence of a large amount of ec-RRF, ec-RRF appears to bind to this site (Kiel et al. 2003).

The corresponding intermediates with the homologous combination are shown as complexes IV, V, and VI, respectively. On the basis of the present finding (Fig. 4), we propose that the critical step during the disassembly of posttermination complexes is the release of RRF concomitant with the release of mRNA by the action of EF-G and GTP (from complex V to VI). This step, when coupled with mRNA release as shown in this figure, is dependent on GTP (Hirashima and Kaji 1973; Hirokawa et al. 2002b). Without mRNA, we have shown that RRF is released by EF-G with GMP-PCP (Kiel et al. 2003) or GTP (Fig. 4).

The scheme described above was derived from studies on disassembly of model post-termination complexes. We believe that the model post-termination complex we use represents a typical post-termination complex except for the absence of the termination codon at the A-site. In contrast to the scheme shown above, with a short mRNA having a strong ribosome-binding sequence, ribosomal subunits are separated by RRF and EF-G before release of mRNA or tRNA from the ribosomes. IF3 is not involved in the formation of subunits from $70 \mathrm{~S}$ ribosomes (Karimi et al. 1999). They showed that IF3 releases tRNA from the complex of the 30S subunit, tRNA, and mRNA in confirmation of the previous report (Gualerzi et al. 1971). In this model, mRNA is not released from ribosomes. The possible reason for the difference was attributed to the sequence of mRNA (Kaji et al. 2001). Indeed, we have observed that the behavior of ribosomes at the termination codon is greatly influenced by the mRNA sequence surrounding the termination codon (Inokuchi et al. 2000). 
A

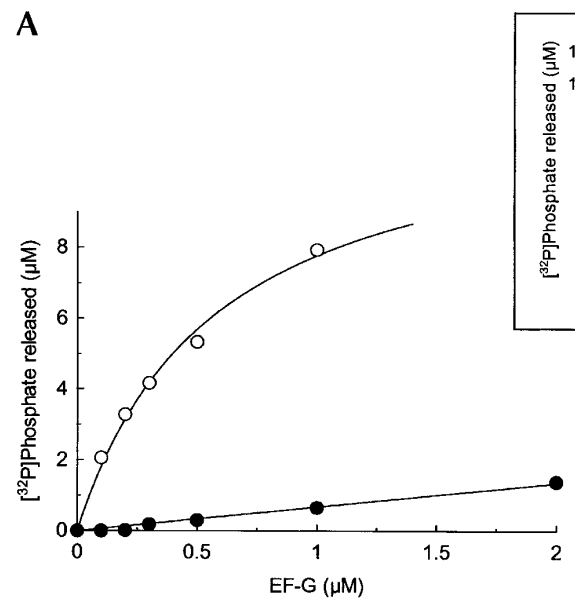

B

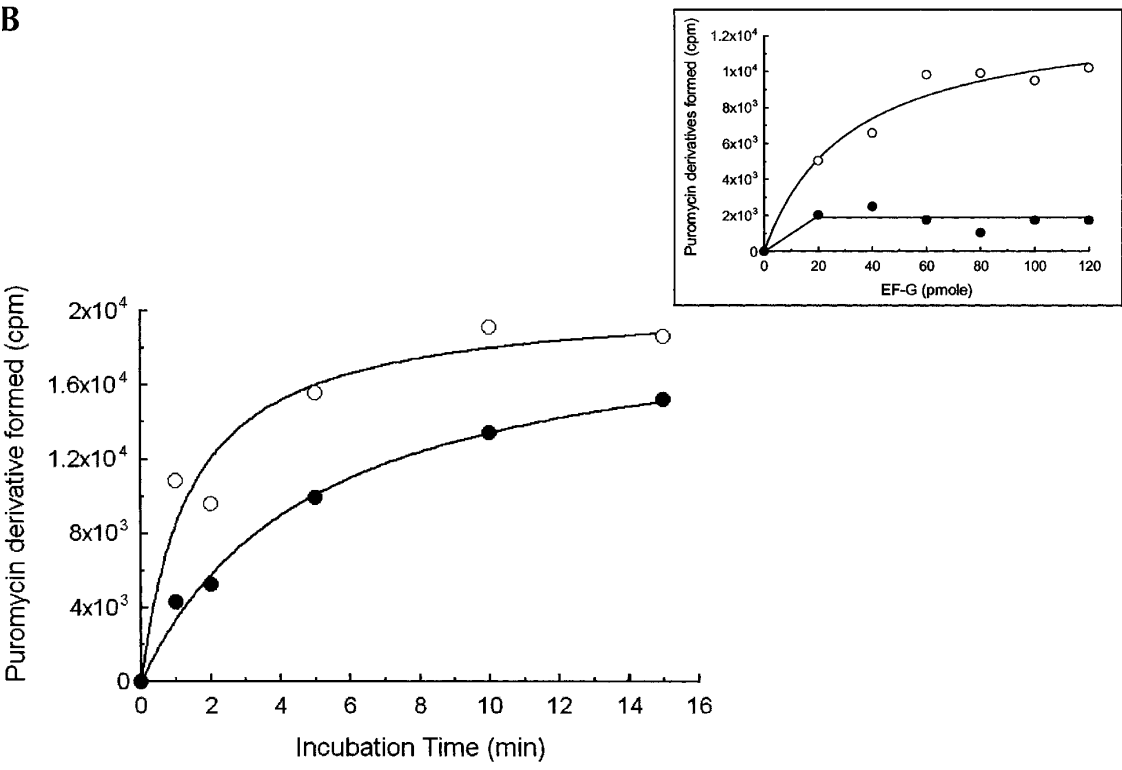

FIGURE 6. GTPase and translocation activities of tt-EF-G with ec-ribosomes. (A) GTP hydrolysis. Dose response curve of EF-G. ${ }^{32} \mathrm{P}$ inorganic phosphate released in 2 min from $50 \mu \mathrm{M}$ GTP, $1.2 \mu \mathrm{Ci}$ of $\left(\gamma^{-32} \mathrm{P}\right)$ GTP upon incubation with $0.2 \mu \mathrm{M}$ ec-ribosomes was estimated as described in Materials and Method. The inset figure shows the dose response curve of ribosomes. It shows the rate per $2 \mathrm{~min}$ in the presence of various concentrations of ribosomes with $0.4 \mu \mathrm{M}$ ec-EF-G or $3 \mu \mathrm{M}$ tt-EF-G. (Open circles) ec-EF-G; (closed circles) tt-EF-G. In the presence of $2 \mu \mathrm{M}$ tt-EF-G, $20,040 \mathrm{cpm}$ of $\left[{ }^{32} \mathrm{P}\right]$ phosphate, corresponding to $1.34 \mu \mathrm{M}\left[{ }^{32} \mathrm{P}\right]$ phosphate, was released. $(B)$ Time course of translocation of peptidyl tRNA. Translocation on ec-ribosomes $(2 \mu \mathrm{M})$ was measured by the formation of puromycin reactive ${ }^{14} \mathrm{C}$-labeled peptidyl tRNA in the presence of $0.2 \mu \mathrm{M}$ of ec- or tt-EF-G at $32^{\circ} \mathrm{C}$ in buffer $\mathrm{R}$ (total volume was $30 \mu \mathrm{L}$ ). Under the experimental conditions, ec-EF-G translocated $0.13 \mu \mathrm{M}$ peptidyl tRNA per $1 \mu \mathrm{M}$ ribosomes in $5 \mathrm{~min}$ (specific activity of ${ }^{14} \mathrm{C}$ phenylalanine was $460 \mathrm{mC} / \mathrm{mmol}$, Amersham, $1200 \mathrm{cpm} / \mathrm{pmol}$ ). The inset figure shows the dose response curve of EF-G with constant amount of ribosome with peptidyl tRNA $(0.12 \mu \mathrm{M})$ at the A/P-site. The incubation period was $5 \mathrm{~min}$. (Open circles) ec-EF-G; (closed circles) tt-EF-G.

In contrast to the report that tt-EF-G does not function with ec-RRF (Ito et al. 2002, see p. 1269, discussion section), we find that tt-EF-G functions well with ec-RRF. Since we are dealing with the identical combinations, E. coli and T. thermophilus, we have no obvious explanation for this discrepancy. The genetic swapping between tt- and ec-factors by Ito et al. led them to conclude that domain I of RRF interacts with domain IV of EF-G. This is in variance with our recent results on the ribosomal binding sites of RRF and EF-G (Wilson and Noller 1998; Agrawal et al. 1999, 2001, 2004; Frank and Agrawal 2000). Our current finding described in this paper explains the reason for the discrepancies. On the other hand, our finding that ec-RRF can work with EF-G from other species is consistent with the report that Mycobacterium tuberculosis EF-G (mtEF-G) can complement an ec-EF-G temperature-sensitive mutant, indicating that mt-EF-G can function with ecRRF for disassembly in vivo (Rao and Varshney 2001). This is not due to RF3 doing the role of EF-G in the RRF reaction because RF3 does not function in place of EF-G for disassembly of model post-termination complexes (data not shown) despite the fact that RF3 substituted EF-G for disassembly of complexes with artificial short nucleotides (Grentzmann et al. 1998). Therefore, tt-EF-G must function with ec-RRF in vivo.

We showed that tt-EF-G functions with ec-ribosomes in vitro although the rate is less than that of ec-EF-G (Fig. 6A,B). The inability of tt-EF-G to complement temperature-sensitive ecEF-G is therefore not due to the inability of tt-EF-G to carry out translocation per se but because the translocation rate is so slow that it is impossible to support the growth of $E$. coli. This is consistent with the notion that in vitro release of tRNA from ec-ribosomes by tt-EF-G and ec-RRF is due to translocation-like activity of EF-G. These observations indicate that in vivo inactivity of foreign proteins or modified proteins may not necessarily indicate that the protein is incapable of doing its function. It could mean that it cannot support the growth of the host for a number of reasons unrelated to the original function of the protein. This is just another possible reason for the discrepancy between our biochemical (Lancaster et al. 2002; Kiel et al. 2003), biophysical data (Agrawal et al. 2004) and conclusions derived from genetic in vivo tests of domain-swapped proteins (Ito et al. 2002; Fujiwara et al. 2004). 


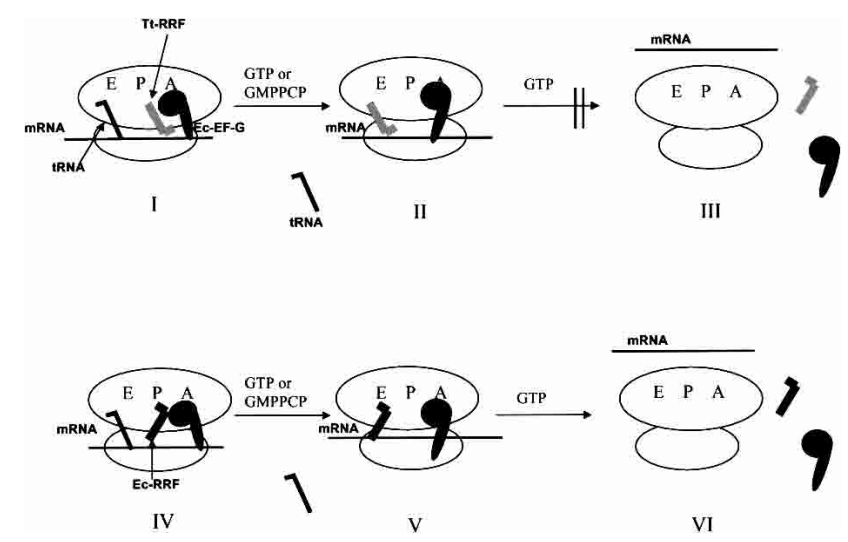

FIGURE 7. Schematic representation of steps of the RRF reaction with ec-ribosomes, tt-or ec-RRF, and ec-EF-G. Complex I is the ecpost-termination complex with tt-RRF (gray) at the A/P-site, ec-EF-G (black) at the pre-translocation site, mRNA (black horizontal line), and tRNA (gray thin hook) at the P/E-site. With the help of either GTP or GMPCP, this is converted to complex II with concomitant release of tRNA. Complex II has tt-RRF at the E-site, ec-EF-G at the post-translocation position, and mRNA. The complex II is the final product in this case and does not proceed further to complex III, vacant ribosome (Fig. 4). Complexes IV and V correspond to complexes I and II, respectively, except that ec-RRF is used. An important difference here is that complex V is converted to complex VI (vacant ribosome without mRNA or RRF).

\section{MATERIALS AND METHODS}

\section{Buffers}

Buffer X contained 10 mM Tris-Cl (pH 7.4), 8.2 mM MgSO 4 , 84 $\mathrm{mM} \mathrm{NH}_{4} \mathrm{Cl}, 1.3 \mathrm{mM}$ DTT, and $50 \mu \mathrm{M}$ puromycin. Binding and release buffer (Buffer R) contained $50 \mathrm{mM}$ Tris- $\mathrm{HCl}$ ( $\mathrm{pH} 7.5), 10$ $\mathrm{mM} \mathrm{Mg}(\mathrm{OAc})_{2}$, and $25 \mathrm{mM} \mathrm{KCl}$.

\section{Ribosomes}

ec-Ribosomes were prepared as described previously (Kiel et al. 2003). tt-Ribosomes were a kind gift from Harry Noller (Univ. of California at Santa Cruz).

\section{ec-RRF and ec-EF-G}

ec-RRF was purified as described (Hirashima and Kaji 1972b). Native ec-EF-G was prepared as described (Hou et al. 1994b). Anti-RRF antibodies were made by Rockland.

\section{tt-RRF and anti-tt-RRF preparation}

The following primers were used to clone the tt-RRF from $T$. thermophilus genomic DNA. The sense primer was NNN-TCTA GAAATAATTTTGTTTAACTTTAAGAAGGAGATATACATATG ACCCTGAAGGAGCTTTACGCG. The anti-sense primer was NNN-GGATCCTCAGCCCAGGATCTCCTGCTCCTT. The PCR DNA was cloned into pGEM-T vector (Promega), and JM 109 (Promega) was transformed by this plasmid. The DNA sequence was confirmed. tt-RRF protein was purified from the strain JM109/pGEM-T/tt-RRF essentially as described by Hirashima and
Kaji (1972b) with added heat treatment. Antiserum was made by Rockland.

\section{tt-EF-G}

The tt-EF-G gene from the tt-EF-G plasmid (pAB98) was placed in XL-10 Gold cells, the transformed cells were grown in LB medium, and tt-EF-G was purified as described (Martemyanov et al. 1998). Less than $1 \%$ of ec-EF-G, if any, was present in the tt-EF-G preparation, as detected by anti-ec-EF-G (data not shown).

\section{RRF assay, disassembly of model post-termination complexes}

The routine assay has been described previously (Hirashima and Kaji 1972a). Details are given in the Figure 1 legend.

\section{Release of tRNA from the post-termination complexes}

The release of tRNA from the model post-termination complexes was examined as described by Hirashima and Kaji (1972a) with some modifications. In brief, polysomes $\left(1 \mathrm{~A}_{260}\right.$ unit) in $550 \mu \mathrm{L}$ of reaction mixture were incubated in $0.5 \mu \mathrm{M}$ ec-EF-G, $363 \mu \mathrm{M}$ GTP, and various concentrations of tt-RRF in Buffer $\mathrm{X}$ at $30^{\circ} \mathrm{C}$ for 15 min. The released tRNA was measured as described (Hirashima and Kaji 1973). Briefly, the released tRNA was separated from ribosomes by the Millipore membrane (pore size $0.45 \mu \mathrm{m}$ ), which traps ribosomes but not tRNA. Released tRNA thus obtained was concentrated by Microcon-10 (centrifuge at 14,000g) and the concentrated tRNA was aminoacylated with $0.15 \mu \mathrm{Ci}$ of $\left[{ }^{14} \mathrm{C}\right]$ amino acid mixture (Amersham). The cold TCA insoluble radioactivity produced was regarded as a mixture of $\left[{ }^{14} \mathrm{C}\right]$ aminoacyl-tRNA formed from unesterified tRNA released from the model posttermination complexes. Assuming that each ribosome of the model post-termination complexes has two unesterified tRNAs (Remme et al. 1989; Stark et al. 1997), 48 pmol of tRNA corresponds to $\sim 7000 \mathrm{cpm}$ under the experimental conditions.

\section{RRF release from the model post-termination complexes and washed ribosomes}

The release of RRF from the model post-termination complexes was measured under the same conditions as mRNA release. The reaction mixture $(275 \mu \mathrm{L})$ containing polysome (0.6 $\mathrm{A}_{260}$ unit) with $0.18 \mu \mathrm{M}$ ec-RRF or tt-RRF, various concentrations of ecEF-G or tt-EF-G, and $0.37 \mathrm{mM}$ GTP in Buffer X was incubated at $30^{\circ} \mathrm{C}$ for $15 \mathrm{~min}$. The bound RRF to the polysomes was isolated by Microcon-100 and estimated using polyclonal antibodies against RRF. The release of RRF from the washed E. coli ribosomes was measured as described by Kiel et al. (2003).

\section{GTPase activity of ec-EF-G and tt-EF-G with ec-ribosomes}

The reaction mixture $(100 \mu \mathrm{L})$ contained $50 \mu \mathrm{M}$ GTP, $1.2 \mu \mathrm{Ci}$ of $\left(\gamma-{ }^{32} \mathrm{P}\right) \mathrm{GTP}$ (Amersham; $\left.\sim 5000 \mathrm{Ci} / \mathrm{mmol}\right)$, various concentrations of ec-ribosomes and ec-EF-G or tt-EF-G in Buffer R as described 
in the legend. The reaction mixture was incubated for $2 \mathrm{~min}$ at $32^{\circ} \mathrm{C}$. An aliquot $(20 \mu \mathrm{L})$ of the reaction mixture was mixed with $50 \mu \mathrm{L}$ of a "stop solution" (0.02 M tungstate, $\left.0.02 \mathrm{~N} \mathrm{H}_{2} \mathrm{SO}_{4}\right), 10$ $\mu \mathrm{L}$ of $2 \mathrm{mM}$ potassium phosphate ( $\mathrm{pH} 6.9$ ), $20 \mu \mathrm{L}$ of $5 \%$ ammonium molybdate in $4 \mathrm{~N} \mathrm{H}_{2} \mathrm{SO}_{4}$. Finally, $100 \mu \mathrm{L}$ of Butanol/Benzene $(1: 1)$ was added to this mixture and thoroughly mixed, and $50 \mu \mathrm{L}$ of the top layer was counted in a scintillation counter (Nishizuka and Lipmann 1966).

\section{Assay of translocation of peptidyl tRNA on ec-ribosomes with ec- or tt-EF-G}

Pre-translocation complexes having peptidyl tRNA on the A/P-site were prepared as follows. A mixture of tRNA ${ }^{\text {Phe }},{ }^{14} \mathrm{C}$-Phe, ATP, and phenylalanyl tRNA synthetase in Buffer $\mathrm{R}$ was incubated at $32^{\circ} \mathrm{C}$ for $20 \mathrm{~min}$. Under this condition, $7451 \mathrm{cpm} / \mathrm{pmol}$ of phetRNA was formed. The total reaction mixture of $80 \mu \mathrm{L}$ was concentrated to $20 \mu \mathrm{L}$ in Microcon-100 at $10,000 \mathrm{~g}$ for $14 \mathrm{~min} .{ }^{14} \mathrm{C}$ phenylalanyl tRNA thus formed was incubated with 3 pmol of ribosomes, poly(U) at $32^{\circ} \mathrm{C}$ for $5 \mathrm{~min}$. Peptidyl tRNA is formed and is bound at the A/P-site at this point (Suzuka and Kaji 1967). Of the total peptidyl tRNA formed, $\sim 50 \%$ was diphenylalanyl tRNA and the rest was oligo-phenylalanyl tRNA. Then GTP, puromycin and either ec-EF-G or tt-EF-G was added and the translocation reaction was continued for another $5 \mathrm{~min}$. The reaction was stopped by the addition of $60 \mu \mathrm{L}$ of ethyl acetate to $20 \mu \mathrm{L}$ of the final reaction mixture. After mixing, the radioactivity of $40 \mu \mathrm{L}$ of the top layer was counted. The A/P-site-bound peptidyl tRNA becomes available for a puromycin reaction after translocation. The oligo-phenylalanyl puromycin formed was measured by ethyl acetate soluble radioactivity (Igarashi et al. 1969). Without EF-G, no significant puromycin derivatives were observed.

\section{ACKNOWLEDGMENTS}

This work was supported by NIH GM 60429 (to A.K.) and the Creative Biomedical Research Institute fund and by Nippon Paint Research Fund (to H.K.). We thank Harry Noller of the University of California, Santa Cruz, for providing us with tt-ribosomes and tt-cells; Mikhail Bubunenko of the NIH and A.T. Kudkov at Pushchino, Russia for a tt-EF-G clone; Mans Ehrenberg of the University of Upsala for RF3; Takuya Ueda of Tokyo University for a clone of phenylalanyl tRNA synthetase; and Michael C. Kiel and Go Hirokawa of the University of Pennsylvania for scientific as well as linguistic advice.

Received October 11, 2004; accepted November 30, 2004.

\section{REFERENCES}

Agrawal, R.K., Heagle, A.B., Penczek, P., Grassucci, R.A., and Frank, J. 1999. EF-G-dependent GTP hydrolysis induces translocation accompanied by large conformational changes in the $70 \mathrm{~S}$ ribosome. Nat. Struct. Biol. 6: 643-647.

Agrawal, R.K., Linde, J., Sengupta, J., Nierhaus, K.H., and Frank, J. 2001. Localization of L11 protein on the ribosome and elucidation of its involvement in EF-G-dependent translocation. J. Mol. Biol. 311: 777-787.

Agrawal, R.K., Sharma, M.R., Kiel, M.C., Hirokawa, G., Booth, T.M., Spahn, C.M.T., Grassucci, R.A., Kaji, A., and Frank, J. 2004.
Visualization of ribosome-recycling factor on the Escherichia coli $70 S$ ribosome: Functional implications. Proc. Natl. Acad. Sci. 101: 8900-8905.

Andersen, L.D., Moreno, J.M.D., Clark, B.F.C., Mortensen, K.K., and Sperling-Petersen, H.U. 1999. Immunochemical determination of cellular content of translation release factor RF4 in Escherichia coli. IUBMB Life 48: 283-286.

Cassataro, J., Velikovsky, C.A., Giambartolomei, G.H., Estein, S., Bruno, L., Cloeckaert, A., Bowden, R.A., Spitz, M., and Fossati, C.A. 2002. Immunogenicity of the Brucella melitensis recombinant ribosome recycling factor-homologous protein and its CDNA. Vaccine 20: 1660-1669.

Frank, J. and Agrawal, R.K. 2000. A ratchet-like inter-subunit reorganization of the ribosome during translocation. Nature 406: 318-322.

Fujiwara, T., Ito, K., Nakayashiki, T., and Nakamura, Y. 1999. Amber mutations in ribosome recycling factors of Escherichia coli and Thermus thermophilus: Evidence for C-terminal modulator element. FEBS Lett. 447: 297-302.

Fujiwara, T., Ito, K., and Nakamura, Y. 2001. Functional mapping of ribosome-contact sites in the ribosome recycling factor: A structural view from a tRNA mimic. RNA 7: 64-70.

Fujiwara, T., Ito, K., Yamami, T., and Nakamura, Y. 2004. Ribosome recycling factor disassembles the post-termination ribosomal complex independent of the ribosomal translocase activity of elongation factor G. Mol. Microbiol. 53: 517-528.

Grentzmann, G., Kelly, P.J., Laalami, S., Shuda, M., Firpo, M.A., Cenatiempo, Y., and Kaji, A. 1998. Release factor RF-3 GTPase activity acts in disassembly of the ribosome termination complex. RNA 4: 973-983.

Gualerzi, C., Pon, C.L., and Kaji, A. 1971. Initiation factor dependent release of aminoacyl-tRNAs from complexes of $30 \mathrm{~S}$ ribosomal subunits, synthetic polynucleotide and aminoacyl tRNA. Biochem. Biophys. Res. Commun. 45: 1312-1319.

Hirashima, A. and Kaji, A. 1972a. Factor-dependent release of ribosomes from messenger RNA-Requirement for two heat-stable factors. J. Mol. Biol. 65: 43-58.

- 1972b. Purification and properties of ribosome-releasing factor. Biochemistry 11: 4037-4044.

- 1973. Role of elongation factor $G$ and a protein factor on the release of ribosomes from messenger ribonucleic acid. J. Biol. Chem. 248: 7580-7587.

Hirokawa, G., Kiel, M.C., Muto, A., Kawai, G., Igarshi, K., Kaji, H., and Kaji, A. 2002a. Binding of ribosome recycling factor to ribosomes-Comparison with tRNA. J. Biol. Chem. 277: 35847-35852.

Hirokawa, G., Kiel, M.C., Muto, A., Selmer, M., Raj, V.S., Liljas, A., Igarashi, K., Kaji, H., and Kaji, A. 2002b. Post-termination complex disassembly by ribosome recycling factor, a functional tRNA mimic. EMBO J. 21: 2272-2281.

Hirokawa, G., Inokuchi, H., Kaji, H., Igarashi, K., and Kaji, A. 2004. In vivo effect of inactivation of ribosome recycling factor-Fate of ribosomes after unscheduled translation downstream of open reading frame. Mol. Microbiol. 10: 1365-2958.

Hou, Y., Lin, Y.-P., Sharer, J.D., and March, P.E. 1994a. In vivo selection of conditional-lethal mutations in the gene encoding elongation factor G of Escherichia coli. J. Bacteriol. 176: 123-129.

Hou, Y., Yaskowiak, E.S., and March, P.E. 1994b. Carboxyl-terminal amino acid residues in elongation factor $\mathrm{G}$ essential for ribosome association and translocation. J. Bacteriol. 176: 7038-7044.

Igarashi, K., Ishitsuka, H., and Kaji, A. 1969. Comparative studies on the mechanism of action of lincomycin, streptomycin and erythromycin. Biochem. Biophys. Res. Commun. 37: 499-504.

Inokuchi, Y., Hirashima, A., Sekine, Y., Janosi, L., and Kaji, A. 2000. Role of ribosome recycling factor (RRF) in translational coupling. EMBO J. 19: 3788-3798.

Ishitsuka, H., Kuriki, Y., and Kaji, A. 1970. Release of transfer ribonucleic acid from ribosomes-A G factor and guanosine triphosphate-dependent reaction. J. Biol. Chem. 245: 3346-3351.

Ito, K., Fujiwara, T., Toyoda, T., and Nakamura, Y. 2002. Elongation factor $\mathrm{G}$ participates in ribosome disassembly by interacting with ribosome recycling factor at their tRNA-mimicry domains. Mol. 
Cell 9: 1263-1272.

Janosi, L., Shimizu, I., and Kaji, A. 1994. Ribosome recycling factor (ribosome releasing factor) is essential for bacterial growth. Proc. Natl. Acad. Sci. 91: 4249-4253.

Janosi, L., Hara, H., Zhang, S., and Kaji, A. 1996. Ribosome recycling by ribosome recycling factor (RRF)-An important but overlooked step of protein biosynthesis. Adv. Biophys. 32: 121-201.

Janosi, L., Mottagui-Tabar, S., Isaksson, L.A., Sekine, Y., Ohtsubo, E., Zhang, S., Goon, S., Nelken, S., Shuda, M., and Kaji, A. 1998. Evidence for in vivo ribosome recycling, the fourth step in protein biosynthesis. EMBO J. 17: 1141-1151.

Janosi, L., Mori, H., Sekine, Y., Abragan, J., Janosi, R., Hirokawa, G., and Kaji, A. 2000. Mutations influencing the frr gene coding for ribosome recycling factor (RRF). J. Mol. Biol. 295: 815-829.

Kaji, A., Kiel, M.C., Hirokawa, G., Muto, A., Inokuchi, Y., and Kaji, H. 2001. The fourth step of protein synthesis: Disassembly of the posttermination complex is catalyzed by elongation factor $\mathrm{G}$ and ribosome recycling factor, a near-perfect mimic of tRNA. Cold Spring Harb. Symp. Quant. Biol. 66: 515-529.

Karimi, R., Pavlov, M.Y., Buckingham, R.H., and Ehrenberg, M. 1999. Novel roles for classical factors at the interface between translation termination and initiation. Mol. Cell 3: 601-609.

Kiel, M.C., Raj, V.S., Kaji, H., and Kaji, A. 2003. Release of ribosomebound ribosome recycling factor by elongation factor G. J. Biol. Chem. 278: 48041-48050.

Kim, K.K., Min, K., and Suh, S.W. 2000. Crystal structure of the ribosome recycling factor from Escherichia coli. EMBO J. 19: 2362-2370.

Lancaster, L., Kiel, M.C., Kaji, A., and Noller, H.F. 2002. Orientation of ribosome recycling factor in the ribosome from directed hydroxyl radical probing. Cell 111: 129-140.

Lowe, A.M., Beattie, D.T., and Deresiewicz, R.L. 1998. Identification of novel staphylococcal virulence genes by in vivo expression technology. Mol. Microbiol. 27: 967-976.

Martemyanov, K.A., Liljas, A., and Gudkov, A.T. 1998. Increased functional activity of elongation factor G with G16V mutation in the GTP-binding domain. Biochemistry (Moscow) 63: 1216-1219.

Moll, I., Hirokawa, G., Kiel, M.C., Kaji, A., and Blasi, U. 2004. Translation initiation with 70 S ribosomes: An alternative pathway for leaderless mRNAs. Nucleic Acids Res. 32: 3354-3363.

Nakano, H., Yoshida, T., Uchiyama, S., Kawachi, M., Matsuo, H., Kato, T., Ohshima, A., Yamaichi, Y., Honda, T., Kato, H., et al. 2003. Structure and binding mode of a ribosome recycling factor (RRF) from mesophilic bacterium. J. Biol. Chem. 278: 3427-3436.

Nishizuka, Y. and Lipmann, F. 1966. Comparison of guanosine triphosphate split and polypeptide synthesis with a purified E. coli system. Proc. Natl. Acad. Sci. 55: 212-219.

Noller, H.F., Yusupov, M.M., Yusupova, G.Z., Baucom, A., and Cate, J.H. 2002. Translocation of tRNA during protein synthesis. FEBS Lett. 514: 11-16.

Rao, A.R. and Varshney, U. 2001. Specific interaction between the ribosome recycling factor and the elongation factor $\mathrm{G}$ from $M y$ cobacterium tuberculosis mediates peptidyl-tRNA release and ribo- some recycling in Escherichia coli. EMBO J. 20: 2977-2986.

Remme, J., Margus, T., Villems, R., and Nierhaus, K.H. 1989. The third ribosomal tRNA-binding site, the $\mathrm{E}$ site, is occupied in native polysomes. Eur. J. Biochem. 183: 281-284.

Rohrback, M.S. and Bodley, J.W. 1976. Steady state kinetic analysis of the mechanism of guanosine triphosphate hydrolysis catalyzed by Escherichia coli elongation factor $\mathrm{G}$ and the ribosome. Biochemistry 15: 4565-4569.

Rolland, N., Janosi, L., Block, M.A., Shuda, A., Teyssier, E., Miege, C., Cheniclet, C., Carde, J., Kaji, A., and Joyard, J. 1999. Plant ribosome recycling factor homologue is a chloroplastic protein and is bactericidal in Escherichia coli carrying temperature-sensitive ribosome recycling factor. Proc. Natl. Acad. Sci. 96: 5464-5469.

Selmer, M., Al-Karadaghi, S., Hirokawa, G., Kaji, A., and Liljas, A. 1999. Crystal structure of Thermotoga maritima ribosome recycling factor: A tRNA mimic. Science 286: 2349-2352.

Seo, H.S., Kiel, M., Pan, D., Raj, V.S., Kaji, A., and Cooperman, B.S. 2004. Kinetics and thermodynamics of RRF, EF-G, and thiostrepton interaction on the Escherichia coli ribosome. Biochemistry 43: $12728-12740$.

Stark, H., Orlova, E.V., Rinke-Appel, J., Junke, N., Mueller, F., Rodnina, M., Wintermeyer, W., Brimacombe, R., and van Heel, M. 1997. Arrangement of tRNAs in pre- and posttranslocational ribosomes revealed by electron cryomicroscopy. Cell 88: 19-28.

Suzuka, K. and Kaji, A. 1967. Studies on diphenylalanine synthesis. Biochim. Biophys. Acta 149: 540-552.

Teixeira-Gomes, A.P., Cloeckaert, A., and Zygmunt, M.S. 2000. Characterization of heat, oxidative, and acid stress responses in Brucella melitensis. Infect. Immun. 68: 2954-2961.

Teyssier, E., Hirokawa, G., Tretiakova, A., Jameson, B., Kaji, A., and Kaji, H. 2003. Temperature sensitive mutation in yeast mitochondrial ribosome recycling factor (RRF). Nucleic Acid Res. 31: 42184226.

Toyoda, T., Tin, O.F., Ito, K., Fujiwara, T., Kumasaka, T., Yamamoto, M., Garber, M.B., and Nakamura, Y. 2000. Crystal structure combined with genetic analysis of the Thermus thermophilus ribosome recycling factor shows that a flexible hinge may act as a functional switch. RNA 6: 1432-1444.

Vizcaino, N., Cloeckaert, A., Dubray, G., and Zygmunt, M. 1996. Cloning, nucleotide sequence, and expression of the gene coding for a ribosome releasing factor-homologous protein of Brucella melitensis. Infect. Immun. 64: 4834-4837.

Wilson, K.S. and Noller, H.F. 1998. Mapping the position of translational elongation factor EF-G in the ribosome by directed hydroxyl radical probing. Cell 92: 131-139.

Yarmolinsky, M.B. and de la Haba, G.L. 1959. Inhibition by puromycin of amino acid incorporation into protein. Biochemistry 45: 1721-1729.

Yoshida, T., Uchiyama, S., Nakano, H., Kashimori, H., Kijima, H., Ohshima, T., Saihara, Y., Ishino, T., Shimahara, H., Yoshida, T., et al. 2001. Solution structure of the ribosome recycling factor from Aquifex aeolicus. Biochemistry 40: 2387-2396. 

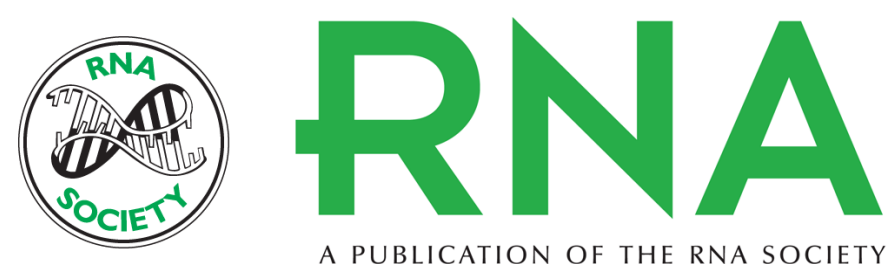

A PUBLICATION OF THE RNA SOCIETY

\section{Interaction of RRF and EF-G from E. coli and T. thermophilus with ribosomes from both origins--insight into the mechanism of the ribosome recycling step}

V. SAMUEL RAJ, HIDEKO KAJI and AKIRA KAJI

RNA 2005 11: 275-284

References This article cites 53 articles, 22 of which can be accessed free at:

http://rnajournal.cshlp.org/content/11/3/275.full.html\#ref-list-1

License

Email Alerting Receive free email alerts when new articles cite this article - sign up in the box at the

Service top right corner of the article or click here.

To subscribe to RNA go to:

http://rnajournal.cshlp.org/subscriptions 